\title{
Effect of temperature on ion motion in future plasma wakefield accelerators
}

\author{
R. Gholizadeh, ${ }^{1}$ T. Katsouleas, ${ }^{2}$ C. Huang, ${ }^{3}$ W. B. Mori, ${ }^{4}$ and P. Muggli ${ }^{1}$ \\ ${ }^{1}$ University of Southern California, Los Angeles, California 90089, USA \\ ${ }^{2}$ Duke University, Durham, North Carolina 27708, USA \\ ${ }^{3}$ Los Alamos National Laboratory, Los Alamos, New Mexico 87545, USA \\ ${ }^{4}$ University of California, Los Angeles, California 90095, USA \\ (Received 3 August 2010; published 16 February 2011)
}

\begin{abstract}
We study the effect of plasma temperature on ion motion in a plasma wakefield accelerator with parameters typical of a future high-energy accelerator. We show that the collapse of the plasma ions caused by the extremely high fields of ultradense electron bunches can be prevented only by a very high plasma ion temperature.
\end{abstract}

DOI: 10.1103/PhysRevSTAB.14.021303

PACS numbers: 52.40.Mj, 52.75.Di, 29.27.-a

Plasmas are capable of sustaining extremely high amplitude electric fields that can be used to accelerate particles to high energies, in distances orders of magnitude shorter than in conventional accelerators. In fact, in a recent experiment at the SLAC National Accelerator Laboratory, the energy of $42 \mathrm{GeV}$ trailing electrons was doubled in only $85 \mathrm{~cm}$ of lithium plasma [1].

In a beam driven plasma-based particle accelerator or plasma wakefield accelerator (PWFA) [2], a high-density relativistic drive electron bunch expels the plasma electrons off its path [Fig. 1(a)] [1]. In order to reach large accelerating fields, the electron bunch must be short, and the plasma density such that the wavelength of the relativistic plasma wave $\lambda_{p e}$ is on the order of the bunch length $\sigma_{z}$ : $\lambda_{p e}=2 \pi c / \omega_{p e} \sim \sigma_{z}$. The plasma angular frequency in a plasma with electron density $n_{e}$ is $\omega_{p e}=\left(2 \pi n_{e} e^{2} / m_{e}\right)^{1 / 2}$. When the beam density $n_{b}$ is larger than the plasma density $\left(n_{b}>n_{e}\right)$, all the plasma electrons are displaced on a time scale given by the electron plasma frequency $\left(\tau_{e} \sim \frac{2 \pi}{\omega_{p e}}\right)$ to a distance on the order of the bunch neutralization radius: $r_{e} \sim\left(n_{b} / n_{e}\right)^{1 / 2} \sigma_{r}$, where $\sigma_{r}$ is the bunch radius. Typically, the ions respond and move on a much longer time scale given by the ion plasma frequency $\left(\tau_{i} \sim \frac{2 \pi}{\omega_{p i}}=\left(\frac{M_{i}}{m_{e}}\right)^{1 / 2} \times\right.$ $\frac{2 \pi}{\omega_{p e}} \gg \frac{2 \pi}{\omega_{p e}} \sim \tau_{e}$ ). Therefore the ions (usually assumed cold, $T_{i}=0$ ) can be considered immobile and the ion column density uniform [Fig. 2(a)] since the wake generation and particle acceleration processes are over after a time $\sim \tau_{e}$. Gauss' law dictates that the uniformly distributed ions create a transverse electric field that increases linearly with radius $r: E_{r}=2 \pi n_{i} e r\left[n_{i}=n_{e}\right.$ for the neutral plasma, Fig. 2(b)], which focuses a following witness bunch. This field also pulls back to the axis the expelled plasma electrons that sustain the accelerating structure [Fig. 1(a)]. The intense longitudinal component of the wakefield accelerates the witness bunch when injected at the proper position behind the drive bunch [Fig. 1(b)]. Energy is therefore transferred from the drive bunch to the witness bunch through the plasma wake.
The ion column also acts as a focusing channel that prevents the two bunches from expanding because of their finite transverse emittances thus allowing for acceleration over long distances $(\sim 1 \mathrm{~m})$ [1]. In the case of a uniform column density with linear focusing force [Figs. 2(a) and 2(b)], the emittance of the witness bunch is preserved upon propagation and acceleration [4]. However, in the case of an ultradense (drive or witness) electron bunch with $n_{b} \gg n_{e}$, the electric field of the bunch is so intense that the cold ions are pulled toward the propagation axis [Fig. 2(c)] on the time scale $\tau_{e}$. This regime is reached when $n_{b} / n_{e}$ approaches $M_{i} / m_{e}$. In this case of ion column collapse, the ion density no longer remains uniform along and across the accelerating structure and the transverse focusing force is nonlinear [Fig. 2(d)]. The nonlinearity in the focusing force leads to emittance growth and thus to degradation of the witness bunch quality [5], which could seriously limit the application of the PWFA to a future plasma-based linear collider. The effect of the nonlinear focusing force has been observed in the symmetric case of a positron bunch propagating in a uniform plasma [6]. Note that emittance growth of the drive bunch itself can be largely ignored as long as it propagates to the end of the plasma acceleration section where it is discarded. The emittance growth of the witness bunch along the plasma must be minimized. In this paper we investigate the possibility of using the plasma temperature to counter the ion column collapse and essentially preserve the bunch emittance.

In a PWFA-based linear collider, a plasma density in the $10^{16}-10^{17} \mathrm{~cm}^{-3}$ range will lead to accelerating fields along the plasma sections in excess of $10 \mathrm{GV} / \mathrm{m}$. At these densities, the size of the accelerating structure is on the order of a few hundred micrometers. Therefore, the drive and witness bunch have to be short to both fit inside the structure. The witness bunch is shorter $(\sim 10 \mu \mathrm{m})$ than the drive bunch $(\sim 35 \mu \mathrm{m})$ in order to minimize its energy spread, which is critical for a linear collider. The drive and witness bunch charge, optimized for efficiency and final 

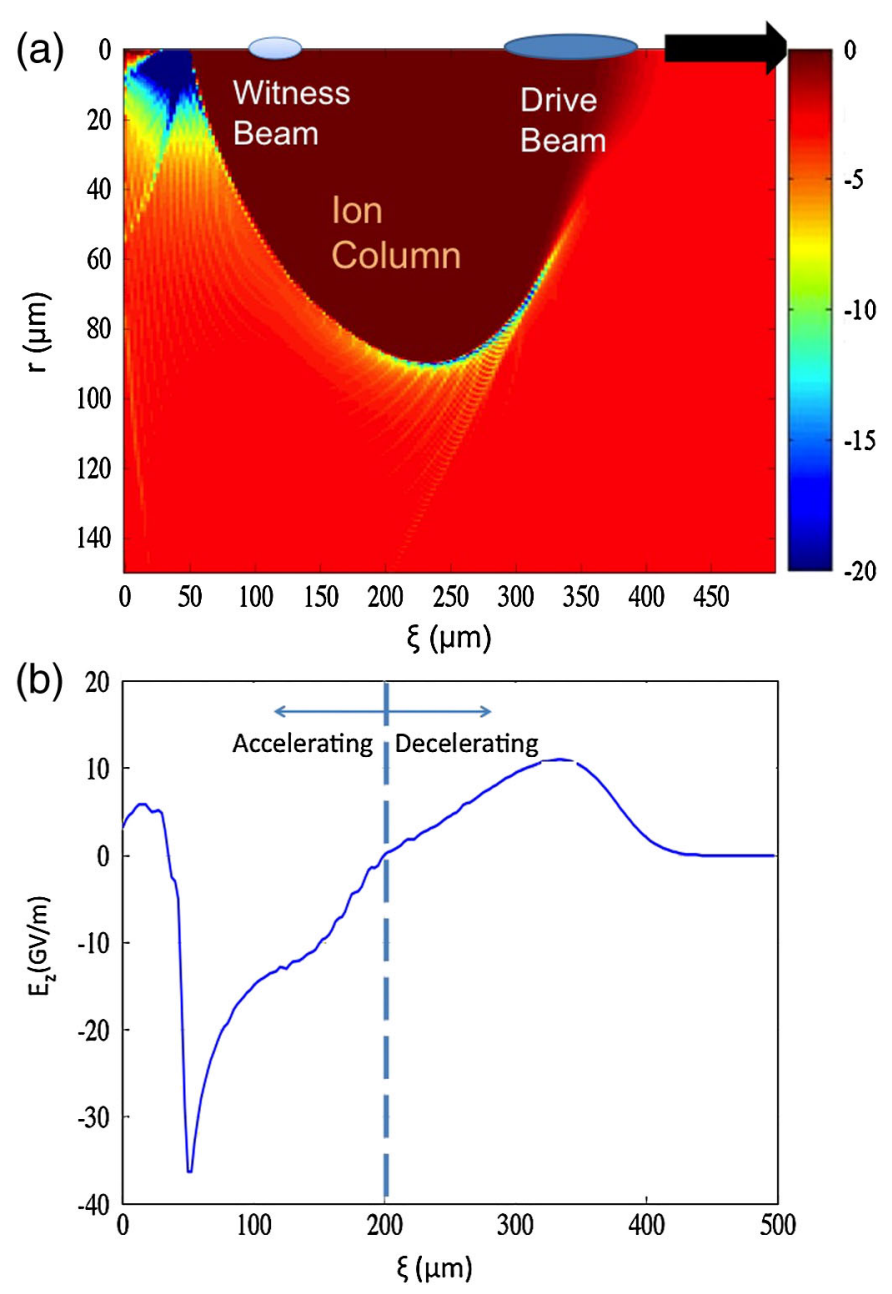

FIG. 1. (a) Density of the plasma electrons obtained from a numerical simulation using the code QUICKPIC [3], showing the pure and (initially) uniform ion column created by the drive bunch moving from left to right. The drive and witness bunch are shown schematically (their transverse sizes are exaggerated for the sake of better visibility). (b) Resulting on-axis longitudinal electric field $E_{z}$ showing that the drive bunch is in the decelerating phase of the wakefield $(\xi \approx 320 \mu \mathrm{m})$ while the witness bunch is placed in the accelerating phase $(\xi \approx 110 \mu \mathrm{m})$. Evidence of accelerating field amplitude flattening in the witness bunch region around $-15 \mathrm{GV} / \mathrm{m}$ is due to the loading of the wakefield. In the pure ion column with no ion motion, the focusing force is linear and the witness bunch incoming emittance is preserved. The parameters are those of Table I. Bunches are $2.5 \sigma_{z}$ long but for the sake of better visibility, bunches transverse sizes are exaggerated. The color bar on (a) indicates $n_{e} / n_{e 0}$ the ratio of the local plasma electron density to the unperturbed plasma density (value of -1 ).

bunch quality, are around $2 \times 10^{10}$ and $0.5 \times 10^{10}$ electrons, respectively. The collider luminosity $\left(\sim 2 \times 10^{34} \mathrm{~s}^{-1} \mathrm{~cm}^{-2}\right)$ calls for extremely small normalized emittances $\left(\gamma \varepsilon_{x}=10\right.$ and $\gamma \varepsilon_{y}=0.04 \mathrm{~mm} \mathrm{mrad}$ at $250 \mathrm{GeV}[5,7])$. In order to minimize synchrotron radiation loss and the production of large amounts of gamma radiation, the bunches transverse size is also small. In particular, (a)
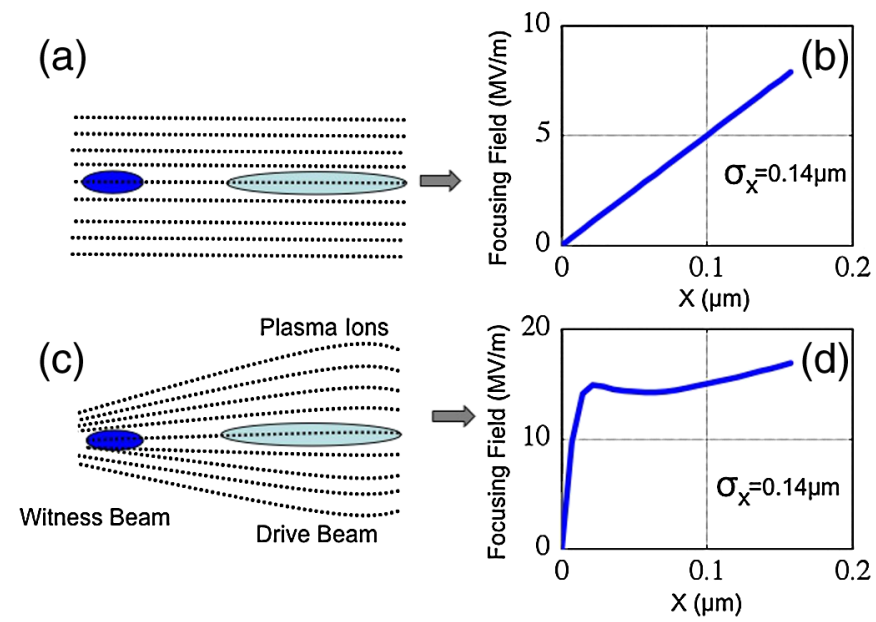

FIG. 2. (a) Schematic of the drive bunch, witness bunch, and ions distribution behind the drive bunch in the case of immobile ions: the ion column density is uniform. (b) Corresponding focusing force at the position of the witness bunch $\left(1.25 \sigma_{z}\right.$ behind the drive bunch peak) calculated for the case of $n_{i}=$ $n_{e}=0.9 \times 10^{16} \mathrm{~cm}^{-3}$. The force is linear over the transverse bunch extent. (c) Schematic for the mobile ions case. (d) Corresponding nonlinear focusing force obtained from simulations showing the resulting radial nonlinearity. Witness bunch transverse size $\sigma_{x}=0.14 \mu \mathrm{m}$.

the witness bunch can be matched to the plasma ion column focusing force $\left\{\sigma_{r}=\left[\left(1 / 2 \pi r_{e}\right)\left(\varepsilon^{2} \gamma / n_{e}\right)\right]^{1 / 4}\right.$, see Eq. (2) below \}, leading to a focused size at the plasma entrance in the submicrometer range. Table I shows the typical parameters of beams in future PWFA-based accelerator. Note that these parameters correspond to a PWFA energy doubling scenarios known as the plasma afterburner [9] with ILC parameters. Other scenarios including using lower energy drive bunches $(\sim 25 \mathrm{GeV})$ and a large number $(\sim 20)$ of meter-long plasma stages are currently also considered. Ion motion [5] is weakly dependent on the beam energy $\left(\sim \gamma^{1 / 4}\right)$ and the ion temperature necessary to counter ion motion is independent of the (relativistic) beam energy.

TABLE I. Typical bunch parameters for a future PWFA-based linear collider adapted from ILC parameters [8].

Drive bunch Witness bunch

\begin{tabular}{lcc}
\hline Train repetition rate $(\mathrm{Hz})$ & 5 & 5 \\
Number of bunches per train & 2820 & 2820 \\
Energy $(\mathrm{GeV})$ & 250 & 250 \\
Number of particles $N_{b}\left(\times 10^{10}\right)$ & 2 & 0.5 \\
Longitudinal size $\sigma_{z}(\mu \mathrm{m})$ & $\sim 35$ & $\sim 10$ \\
Transverse size $\sigma_{r}(\mu \mathrm{m})$ & 0.14 & 0.14 \\
Normalized emittance $x$ & & \\
$\gamma \varepsilon_{x}(\mathrm{~mm}$ mrad) & 10 & 10 \\
Normalized emittance $y$ & & \\
$\gamma \varepsilon_{y}(\mathrm{~mm}$ mrad) & 0.4 & 0.4 \\
\hline \hline
\end{tabular}


Several techniques have already been proposed to mitigate the ion motion or minimize the emittance growth $[10,11]$. In this paper, we investigate the possibility of using plasma ions with a nonzero temperature, to counter the strong force imposed on them by the dense electron bunches.

The international linear collider (ILC) design requires a repetition rate of the incoming beam train of $5 \mathrm{~Hz}$, each train containing 2820 bunches $[7,8]$ to reach the luminosity requirement for physics discoveries. Considering a PWFAbased version of a linear collider with parameters similar to those of the ILC (Table I), this means that on average a new bunch pair enters the plasma every 337 ns. Each drive bunch deposits a large amount of its energy, on the order of $10 \%$ for a high efficiency optimized system, causing the plasma temperature to rise significantly along the train. The plasma can be considered as a gas with pressure proportional to its temperature (in thermal equilibrium), and hotter plasma with higher pressure offers a greater resistance against the ion column collapse. This effect could be useful to counter the plasma ion motion. Although it may be difficult to estimate the plasma temperature at a given beam repetition rate, it is useful to study the dependency of ion collapse with respect to plasma ion temperature. In this paper, we look at the equations for the plasma ion motion as a function of temperature in a simplified model. We find analytically the temperature that is required to fully eliminate the ion motion and verify the results using particle-in-cell (PIC) simulations.

To investigate the effect of temperature on the collapse of the plasma ion column caused by the large transverse space charge field of a single drive or witness electron bunch, we describe the plasma ions inside the electron bunch as an "ion beam" with no longitudinal momentum in the laboratory frame and with a transverse "emittance" given by the ion temperature. We emphasize that the ion motion resulting from the electron bunch electric field is a bulk motion, whereas increasing the ion temperature leads to random motion of the ions. In the electron beam frame, the ion beam is moving backward with the electron beam velocity close to the speed of light. The behavior of the plasma ions upon the passage of the electron bunch is described by an envelope equation for the "ion beam" with transverse rms size $\sigma_{r}$ (not necessarily Gaussian). The envelope equation [12] for the plasma ions, moving in the opposite direction of the beam is

$$
\frac{d^{2} \sigma_{r}}{d \xi^{2}}+K_{b} \sigma_{r}=\frac{\varepsilon_{i}^{2}}{\sigma_{r}^{3}}
$$

in which $\varepsilon_{i}$ is the "emittance of the ion beam" related to the ion temperature, $\xi$ is the distance measured in the beam Galilean frame, and $K_{b}=F_{r} /(\gamma m r)$ is the focusing strength exerted by the electron bunch space charge field on the ions and is responsible for the ion motion and column collapse. This equation is valid near the center of the bunch in $r$ and $\xi$, where the bunch density can be approximated as constant. For the sake of simplicity, we assume that the electron beam transverse density $n_{b}$ is uniform and therefore $K_{b}$ has an expression similar to that of a uniform ion column: $K_{b}=2 \pi n_{b} e^{2} / \gamma_{i} M_{i} c^{2}$, where $M_{i}$ is the plasma ion mass and $\gamma_{i}=1$ for the nonrelativistic ions. In order for the ions to remain immobile, the ions pressure force must be equal and opposite to the focusing force of the electron bunch. The equilibrium is calculated by setting $d^{2} \sigma_{r} / d \xi^{2}=0$ in Eq. (1), which results in

$$
K_{b} \sigma_{r}^{4}=\varepsilon_{i}^{2} .
$$

This is similar to the matching condition for an electron beam and the focusing force of a uniform density column of immobile ions. The emittance of the ion beam (at a waist) with temperature $T_{i}$ is

$$
\varepsilon_{i}=\sigma_{r} \frac{v_{x}}{c}=\frac{\sigma_{r}}{c} \sqrt{\frac{k_{B} T_{i}}{M_{i}}},
$$

in which the Boltzmann formula $M_{i} v_{x}^{2}=k_{B} T_{i}$ is used to relate the transverse thermal velocity of the ions $v_{r}$ to the plasma ion temperature with 2 degrees of freedom. Inserting Eq. (3) into Eq. (2) and using $n_{b}=N_{b} /$ $(2 \pi)^{3 / 2} \sigma_{r}^{2} \sigma_{z}$ for a bunch with $N_{b}$ electrons leads to

$$
T_{0}=\frac{N_{b} e^{2}}{\sqrt{2 \pi} k_{B} \sigma_{z}} .
$$

It is interesting to note that the equilibrium ion temperature is independent of the ion mass and of the beam energy. Inserting typical values for a linear collider bunch, $N_{b}=$ $2 \times 10^{10}$ and $\sigma_{z}=35 \mu \mathrm{m}$ results in $T_{0} \sim 4 \times 10^{9} \mathrm{~K} \sim$ $3.4 \times 10^{5} \mathrm{eV}$, an extremely high temperature, probably impractical for a performed plasma.

A somewhat lower temperature may be sufficient to decrease the emittance growth associated with the ion column collapse. Upon partial collapse of the ion column, the plasma temperature rises, resulting in an increase in pressure and further countering the bunch focusing force. Once the plasma temperature reaches that of the equilibrium state, further collapse is prevented. To investigate this effect, we start by treating the plasma as an ideal gas experiencing adiabatic compression. Therefore, we assume that the ion gas follows the following equations of state:

$$
\begin{gathered}
P V=N K T \\
P V^{\Gamma}=\mathrm{const},
\end{gathered}
$$

in which $\Gamma=(f+2) / f$ and $f$ is the degrees of freedom that is assumed to be two in this case, since we only consider the transverse motion of the plasma ions. Using Eqs. (5) and (6) and the fact that the volume $V$ is proportional to $\sigma_{r}^{2}$ (cylindrical coordinates), 


$$
\sigma_{r}^{2(\Gamma-1)} T=\sigma_{r_{0}}^{2(\Gamma-1)} T_{0}=\text { const. }
$$

The ion emittance as a function of $\sigma_{r}$ is then

$$
\varepsilon_{i}=\frac{\sigma_{r}}{c} \sqrt{\frac{k_{B} T}{M_{i}}}=\frac{\sigma_{r_{0}}^{\Gamma-1}}{c \sigma_{r}^{\Gamma-2}} \sqrt{\frac{k_{B} T_{0}}{M_{i}}},
$$

where $\sigma_{r 0}$ is the initial bunch size. It increases as the mismatched ion "beam" pinches under the action of the electron bunch focusing field and the ion temperature increases. The matching condition (2) for this case becomes

$$
T_{0}=\frac{N_{b} e^{2}}{\sqrt{2 \pi} k_{B} \sigma_{z}}\left(\frac{\sigma_{r_{f}}^{2}}{\sigma_{r_{0}}^{2}}\right)^{\Gamma-1}
$$

where $\sigma_{\text {rf }}$ is the final bunch size. Comparing Eq. (9) with Eq. (4) shows a factor of $\left(\sigma_{\mathrm{rf}}^{2} / \sigma_{r 0}\right)^{\Gamma-1}$ reduction in initial temperature is obtained. Since the ion density in cylindrical coordinates scales with $1 / \sigma_{r}{ }^{2}$, the reduction factor is $\left(n_{0} / n_{f}\right)^{1 / 2}$, namely, the square root of the ratio of the initial ion density to the final ion column density. For instance, allowing the ion density to rise by a factor of 9, i.e., the ion column and ion beam radius by a factor of 3 , results in an initial temperature of $T_{0} \sim 1.1 \times 10^{5} \mathrm{eV}$, lower than that for no ion collapse at all, but still rather high.

In order to verify the results of the analytical equations, we perform a simulation run using reduced algorithm PIC code QUICKPIC [3]. In these simulations, a drive bunch with longitudinal size $\sigma_{z}=35 \mu \mathrm{m}$, number of particles $N_{b}=$ $2 \times 10^{10}$, and normalized emittance $0.1 \mathrm{~mm}$ mrad is sent into a hydrogen plasma with various ion temperatures. The plasma ion density is measured on axis along the bunch. Here we consider the ion motion created by the drive bunch only. We assume that the witness bunch is shorter and carries less charge than the drive bunch. The degree of ion column collapse is proportional to $\left(N_{b} \sigma_{z}\right)^{1 / 2}$ [5] and it is reasonable to assume that the witness bunch does not create significant ion motion on its own. It is however affected by the ion column collapse created by the preceding witness bunch. Figure 3 shows the resulting on-axis ion density along the bunch moving to the right, obtained from simulations with different initial ion temperatures. Since the ions are no longer pulled in once the drive bunch passes, but rather repelled by their own space charge, the maximum collapse occurs at the tail of the drive beam. Therefore, we assume that the witness bunch experiences the same focusing force as that at the tail of the drive bunch (i.e. at $\xi=0$ or $1.25 \sigma_{z}$ after the peak of the drive bunch). Figure 4 shows the corresponding radial focusing forces at the same location, measured after the bunch reaches equilibrium along the plasma (reached at $z>20 \mathrm{~cm}$ in Fig. 5). With an initial temperature of $3.4 \times 10^{5} \mathrm{eV}$, the focusing force is practically the same as that in the plasma with immobile ions, clearly showing that high initial temperature can suppress the effect of ion motion. It is worth noticing that at a temperature of $2 \times 10^{5} \mathrm{eV}$, the focusing

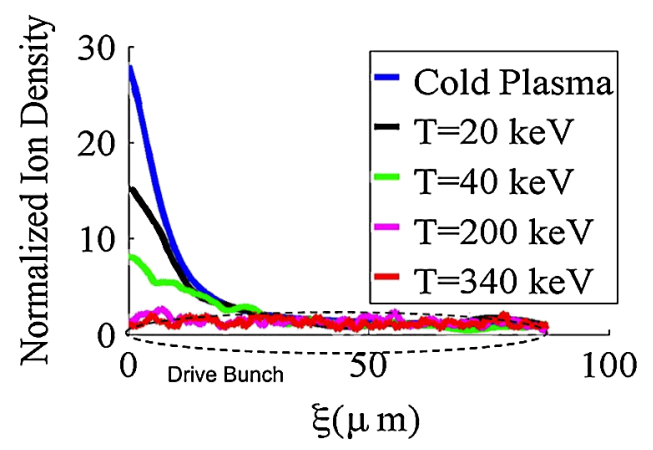

FIG. 3. On-axis plasma ion densities along drive bunch $\left(\sigma_{z}=\right.$ $35 \mu \mathrm{m})$ traveling to the right for various plasma ion temperatures obtained from simulations with mobile ions after $40 \mathrm{~cm}$ of plasma. The ion density in the back of the bunch decreases to the immobile ion value as the plasma ion temperature increases. Initial plasma density is $n_{e}=0.9 \times 10^{16} \mathrm{~cm}^{-3}$. The $\pm 1.25 \sigma_{z}$ contour of the beam is shown schematically. Effective length of the beam is assumed $2.5 \sigma_{z}$.

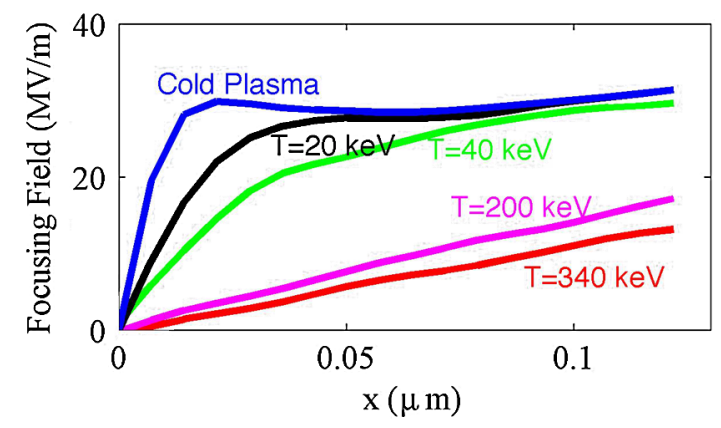

FIG. 4. Radial focusing force on the tail of the drive bunch $\left(1.25 \sigma_{z}\right.$ from the peak or $\xi=0 \mu \mathrm{m}$ on Fig. 3) for different plasma ion temperatures after $20 \mathrm{~cm}$ of plasma. The transverse bunch size is $\sigma_{r}=0.14 \mu \mathrm{m}$. The focusing field becomes more linear (closer to the immobile ion case, not shown) with increasing ion temperature.

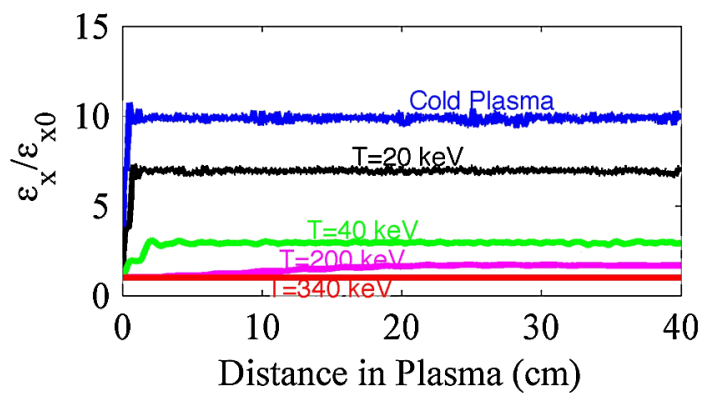

FIG. 5. Relative emittance growths $\left(\varepsilon_{x} / \varepsilon_{x 0}, \varepsilon_{x 0}=0.1 \mathrm{mmmrad}\right.$ incoming emittance) along the plasma for the various plasma ion temperatures of Figs. 3 and 4.

force is still linear, but with a slightly higher slope, i.e., corresponding to a stronger focusing force than in the hotter ion plasma or in the no ion motion case. At temperatures below that, the focusing force is no longer linear 
and the nonlinearity in the focusing force increases with lower ion temperatures, as expected.

Figure 5 shows the beam emittance evolution along the plasma (normalized to the incoming emittance) for a beam evolving in the focusing fields self-consistently calculated at the various ion temperatures of Figs. 3 and 4. The emittance is the rms value calculated from the particles position and momentum values obtained from the simulations. The highly nonlinear focusing force in the cold plasma with mobile ions leads to a very rapid emittance growth of a factor of 10 over the first few millimeters of plasma. Then the emittance remains constant at this high value. The emittance growth for plasma temperatures of $2 \times 10^{5} \mathrm{eV}$ and above is very small $(<10 \%)$. This is in agreement with the results of Fig. 4 that show that the focusing forces is linear, as in the immobile ions case for which emittance is preserved (not shown).

It is important to note that high plasma temperatures give rise to a number of other effects. First, such high temperature plasmas would be very difficult to confine even for short times. Second, high temperature plasma electrons (with temperature similar to that of the ions) can be more easily trapped even though the plasma wave is ultrarelativistic [13,14]. Simple estimates show that the trapping energy threshold for plasma electrons is in the few $\mathrm{MeV}$ range [15], i.e., not much higher than the temperatures necessary to suppress the effect of ion motion. This deleterious effect is not included in the model and simulations. Preliminary results also indicate that such high temperatures do not significantly affect the wakefield structure. However, it is clear that ion temperatures that can prevent ion motion are too high to be used in practical cases.

Finally, we note that Eq. (6) in Ref. [4] suggests that the amount of ion motion can be reduced by shortening the drive bunch length $\left(\sigma_{z}\right)$. However, the bunch lengths considered here are already extremely short. In addition, to preserve the emittance of the witness bunch following the drive bunch, the ion motion over the accelerating structure length $\left(\sim \lambda_{p e}\right)$ must be minimized, not over $\sigma_{z}$ as implied in Ref. [4] for the case of a single bunch.

In conclusion, we have studied the effect of an initial plasma ion temperature (random motion) on the bulk motion of these ions, under the influence of the extremely large electric field of an electron bunch with parameters typical of a future high-energy PWFA-based collider. We showed through calculations and simulations that, with ion temperatures in the few hundred $\mathrm{keV}$ range, the plasma ion motion is significantly reduced and the emittance growth substantially suppressed. In this case, the ion focusing force remains linear with radius and the electron beam emittance can in principle be preserved. High plasma temperatures may naturally arise from the amount of energy deposited in the plasma by the high repetition rate, high power PWFA drive bunches. However, if the plasma reaches such high temperatures, plasma confinements, plasma particle trapping, and its impact on the wakefield structure are issues that have to be taken into account.

\section{ACKNOWLEDGMENTS}

The authors would like to thank USC High Performance Computations \&amp; Communications (HPCC). This work is supported by Department of Energy Contract No. DE-FG02-92ER40745.

[1] I. Blumenfeld et al., Nature (London) 445, 741 (2007).

[2] P. Chen et al., Phys. Rev. Lett. 54, 693 (1985).

[3] C. H. Huang et al., J. Comput. Phys. 217, 658 (2006).

[4] P. Muggli et al., Phys. Rev. Lett. 93, 014802 (2004); C. E. Clayton et al., Phys. Rev. Lett. 88, 154801 (2002).

[5] J. B. Rosenzweig et al., Phys. Rev. Lett. 95, 195002 (2005).

[6] P. Muggli et al., Phys. Rev. Lett. 101, 055001 (2008).

[7] ILC GDE, SBE2009Proposal Document, edited by M. Ross, N. Walker, and A. Yamamoto (2009), http://lcdev .kek.jp/SB20091217B.pdf.

[8] T.O. Raubenheimer, in 11th Advanced Accelerator Concepts Workshop, AIP Conf. Proc. No. 737 (AIP, New York, 2004), p. 86.

[9] S. Lee et al., Phys. Rev. ST Accel. Beams 5, 121301 (2002).

[10] R. Gholizadeh et al., in Proceedings of the 2007 Particle Accelerator Conference, Albuquerque, New Mexico (IEEE, Albuquerque, New Mexico, 2007).

[11] R. Gholizadeh et al., Phys. Rev. Lett. 104, 155001 (2010).

[12] M. Conte and W.W. McKay, An Introduction to the Physics of Particle Accelerators (World Scientific, Singapore, 2008), 2nd ed.

[13] A. I. Akhiezer and R. P. Plovin, Sov. Phys. JETP 3, 696 (1956).

[14] W. B. Mori and T.C. Katsouleas, Phys. Scr. T30, 127 (1990).

[15] T. Katsouleas and J.M. Dawson, UCLA Report No. IPFRPPG- 1121, 1988. 